\title{
Ligand Conversion in Nanocrystal Synthesis: The Oxidation of Al- kylamines to Fatty Acids by Nitrate
}

\author{
Mariano Calcabrini, Dietger Van den Eynden ${ }^{\dagger}$, Sergi Sánchez Ribot, Rohan Pokratath ${ }^{\dagger}$, Jordi Llorca \\ Jonathan De Roo ${ }^{*}$, Maria Ibáñez*
}

IST Austria, Am Campus 1, 3400 Klosterneuburg, Austria.

\begin{abstract}
Ligands are a fundamental part of nanocrystals. They control and direct nanocrystal syntheses, and provide colloidal stability. Bound ligands also affect the nanocrystals' chemical reactivity and electronic structure. Surface chemistry is thus crucial to understand nanocrystal properties and functionality. Here, we investigate the synthesis of metal oxide nanocrystals $\left(\mathrm{CeO}_{2-\mathrm{x}}, \mathrm{ZnO}\right.$, and $\left.\mathrm{NiO}\right)$ from metal nitrate precursors, in the presence of oleylamine ligands. Surprisingly, the nanocrystals are capped exclusively with a fatty acid instead of oleylamine. Analysis of the reaction mixtures with nuclear magnetic resonance spectroscopy revealed several reaction byproducts and intermediates that are common to the decomposition of $\mathrm{Ce}, \mathrm{Zn}, \mathrm{Ni}$ and $\mathrm{Zr}$ nitrate precursors. Our evidence supports the oxidation of alkylamine and formation of a carboxylic acid, thus unraveling this counterintuitive surface chemistry.
\end{abstract}

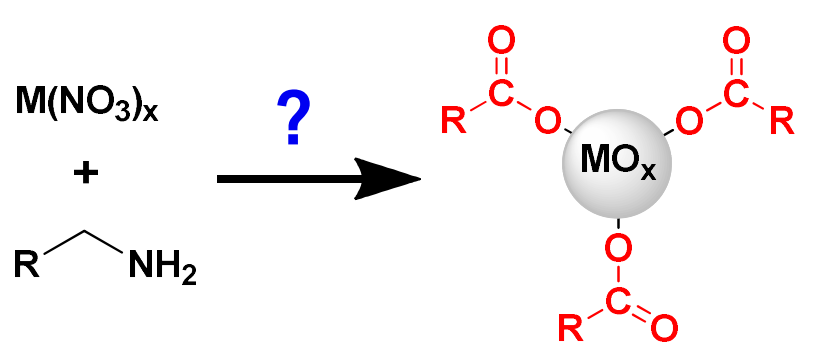

Ligand-assisted syntheses allow preparing colloidally stable nanocrystals (NCs) with controlled size, ${ }^{1-4}$ shape,${ }^{5-8}$ and composition. ${ }^{9-11}$ In these syntheses, long-chain aliphatic ligands are used to dissolve the precursors and control nucleation and growth. ${ }^{12,13}$ After the syntheses, some ligands remain bound to the $\mathrm{NC}$ surface providing colloidal stability and determining solubility, ${ }^{14,15}$ reactivity, ${ }^{16-20}$ and electronic structure. ${ }^{21-25}$ Therefore, unveiling the structure and binding motif of surface ligands is fundamental to understand NC properties, ${ }^{26}$ design ligand exchange strategies, ${ }^{20,27-30}$ and envision potential applications. ${ }^{31,32}$

In reactions where several organic ligands can bind to the NCs, the surface chemistry is typically studied in detail by nuclear magnetic resonance (NMR) spectroscopy, e.g., for CdSe, ${ }^{28,33} \mathrm{PbS},{ }^{34-36}$ and $\mathrm{InP}^{29,37} \mathrm{NCs}$. However, in reactions with only one type of ligand, the NC surface is often assumed to be capped by this particular ligand. Previously, this assumption was proven wrong, with trioctylphosphine oxide decomposing to phosphinic and phosphonic acid ligands during the synthesis of several metal oxides. ${ }^{38}$

Here, we disclose an even more extreme example, where alkylamine ligands are oxidized into carboxylic acids during the synthesis of $\mathrm{CeO}_{2-\mathrm{x}} \mathrm{NCs}$ from cerium nitrate. Investigating the intermediates and reaction byproducts using various NMR techniques, we propose a reaction path and cross-examine it with rigorous control experiments. We further analyzed the synthesis of other oxide NCs ( $\mathrm{NiO}$ and $\mathrm{ZnO}$ ) from the corresponding nitrates and found that the amine undergoes the same reactions.

In a typical synthesis, $\mathrm{Ce}\left(\mathrm{NO}_{3}\right)_{3} \cdot 6 \mathrm{H}_{2} \mathrm{O}$ is dissolved under vacuum in oleylamine and 1-octadecene forming a complex, $\left[\mathrm{Ce}\left(\mathrm{RNH}_{2}\right)_{\mathrm{n}}\left(\mathrm{NO}_{3}\right)_{3}\right]^{5,39}$ This complex thermally decomposes, yielding $\mathrm{CeO}_{2-\mathrm{x}} \mathrm{NCs}$.

$$
\begin{gathered}
\mathrm{Ce}\left(\mathrm{NO}_{3}\right)_{3} \cdot 6 \mathrm{H}_{2} \mathrm{O}+n \mathrm{RNH}_{2} \stackrel{200^{\circ} \mathrm{C}}{\longrightarrow}\left[\mathrm{Ce}\left(\mathrm{NH}_{2} \mathrm{R}\right)_{n}\left(\mathrm{NO}_{3}\right)_{3}\right] \\
{\left[\mathrm{Ce}\left(\mathrm{NH}_{2} \mathrm{R}\right)_{n}\left(\mathrm{NO}_{3}\right)_{3}\right] \stackrel{300^{\circ} \mathrm{C}}{\longrightarrow} \mathrm{CeO}_{2-x}+\mathrm{N}_{2}+\mathrm{NO}_{x}+?}
\end{gathered}
$$

We use here $n$-octadecane instead of 1-octadecene, due the latter's tendency for spontaneous polymerization. ${ }^{40}$ The purified NCs have a quasi-spherical shape, an average crystallite size of $6.5 \mathrm{~nm}$, and a cubic crystallographic phase with space group Fm-3m (Figure S1, S24 and S25)..$^{5,41}$ Using X-ray photoemission spectroscopy we determined that the particles have a composition $\mathrm{CeO}_{1.74}$ (Table S3). Furthermore, we performed thermogravimetric analysis of the NCs and found $15 \%$ wt. organics, corresponding to a ligand coverage of 3.3 ligands $/ \mathrm{nm}^{2}$ (Figure S3), consistent with values reported for other oxide NCs. ${ }^{14,42}$ The broad resonances in the ${ }^{1} \mathrm{H}$ NMR spectrum of the NCs indicate bound ligands (Figure 1). ${ }^{43}$ However, the broadening also prevents their identification. To overcome this limitation, we treated the NCs with trifluoroacetic acid, which strips the original ligands from the surface. ${ }^{5,10}$ The stripped ligands exhibit sharp resonances consistent with the fingerprint of a fatty acid (Figure 1). Especially the $\boldsymbol{\alpha}$ resonance is diagnostic, aligning well with the $\boldsymbol{\alpha}$ resonance of oleic acid $(2.4 \mathrm{ppm})$ and clearly different from the $\boldsymbol{\alpha}$ resonance of protonated oleylamine (2.7 ppm, Figure S4). The alkene resonance $\varepsilon$ of the fatty acid reveals a mixture of cis and trans isomers, similar to that observed in commercial oleylamine, ${ }^{44}$ suggesting that the acid is formed from the amine (Figure S4). It should be noted that the integral of the alkene resonance is lower than expected, pointing to additional reactions concerning the double bond (Figure S5).

We set out to investigate the reaction path leading to the formation of the carboxylic acid. To avoid the reactivity of the double 
bond, we replaced oleylamine with hexadecylamine. This also simplifies the NMR spectra since hexadeylamine has no alkene resonance. The synthesis with hexadecylamine yields NCs with similar characteristics (size, shape, crystal structure, Figure S2). Also the NC surface is again capped by a fatty acid, in this case palmitic acid (hexadecanoic acid) (Figure S6).

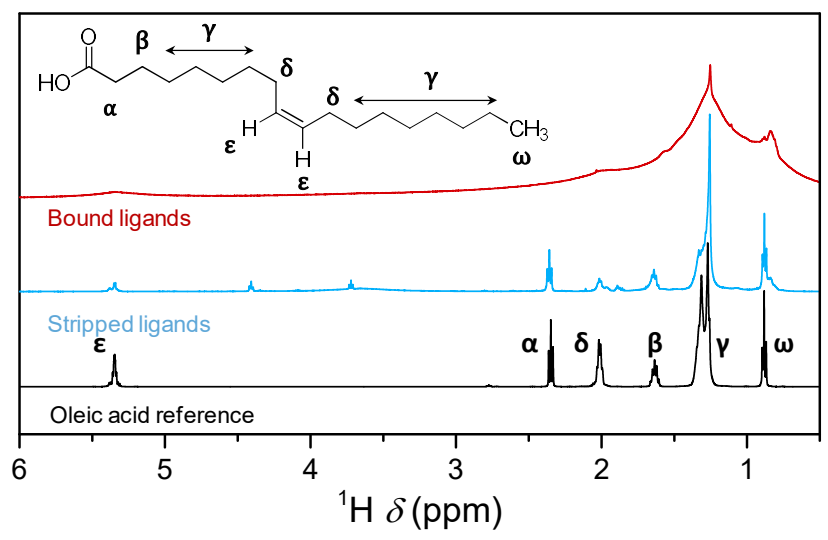

Figure 1. ${ }^{1} \mathrm{H}$ NMR spectra of purified NCs in benzene- $\mathrm{d}_{6}$, the stripped ligands in $\mathrm{CDCl}_{3}$ and the oleic acid reference in $\mathrm{CDCl}_{3}$ with additional trifluoroacetic acid added.

Figure 2 shows the ${ }^{1} \mathrm{H}$ NMR spectrum of the reaction mixture after completion of the synthesis. Besides the starting alkylamine and the solvent, twelve additional resonances are present in the ${ }^{1} \mathrm{H}$ NMR spectrum, labeled $A$ to $M$ in Figure 2. Using advanced NMR spectroscopy, we assigned the resonances to six different compounds derived from hexadecylamine. The reaction mixture contains a secondary aldimine (6, resonances $A, G$ and $K)$, a terminal alkene (2, resonances $B, E$ and $M)$, an amide $(\mathbf{9}$, resonances $D, H$ and $L$ ), an alcohol $(\mathbf{3}$, resonance $F$ ), a nitrile $(\mathbf{5}, J)$ and yet unidentified compounds (resonances $C, I$ ). The identification of these compounds was further confirmed by adding either commercial or synthesized reference compounds to the reaction mixture (spiking experiments). The road map for the assignments, together with the complete data set, can be found in the SI.

Scheme 1 illustrates our proposed reaction path from the alkylamine ligand into the different byproducts and intermediates. The different molecules are labeled $\mathbf{1 - 9}$, and color-coded to indicate how these were identified. The amine 1 reacts with nitrates in different ways. The alkene $\mathbf{2}$ and alcohol $\mathbf{3}$ and are expected products of the reaction of alkylamines with nitrate, as they have been reported for the decomposition of methylamine and ethylamine copper (II) nitrate complexes. ${ }^{45}$ The high reaction temperature might also promote the formation of the alkene 2 by $\beta$-elimination of the amine 1 under basic conditions. ${ }^{46}$

The formation of the other identified byproducts involves the oxidation of the amine group. For the formation of the secondary aldimine $\mathbf{6}$ and the nitrile $\mathbf{5}$, we proposed an intermediate: the primary aldimine 4 . We hypothesize that $\mathbf{1}$ is oxidized to $\mathbf{4}$ by nitrate. Then, $\mathbf{4}$ can be further oxidized to the nitrile $\mathbf{5}$, which is observed in the reaction mixture. ${ }^{47}$ Furthermore, the primary aldimine 4 can condense with a second equivalent of amine $\mathbf{1}$ forming the more stable secondary aldimine $\mathbf{6 . 4 8 , 4 9}$ Aldimine $\mathbf{6}$ is observed in the reaction mixture and is also a common byproduct of the synthesis of nitrides. $^{50}$ The formation of $\mathbf{6}$ entails the evolution of ammonia, which we detected in the gas phase (see SI p. S16). The intermediate 4 can not detected in the reaction mixture (Figure 2), which we attribute to the low stability of primary aldimines. ${ }^{51}$ For the formation of the carboxylic acid $\mathbf{8}$ and the amide $\mathbf{9}$, we propose aldehyde 7 as intermediate. This aldehyde is the oxidation product of the alcohol $3,45,52$ or could be obtained by hydrolysis of either aldimine with adventitious water. ${ }^{51}$ The aldehyde 7 is further oxidized to the carboxylic acid $\mathbf{8}$. Finally, the carboxylic acid $\mathbf{8}$ either binds to the NC surface or condenses with amine $\mathbf{1}$ to form amide 9, explaining the absence of free carboxylic acid in the mixture. ${ }^{53}$ Aldehyde 7 was not detected in the mixture but it appears as a logical intermediate. At the reaction temperature, it easily reacts with amine $\mathbf{1}$, forming the secondary aldimine $\mathbf{6}$.

Further insights into the chemical transformations were obtained by analyzing aliquots of the reaction mixture taken at different reaction temperatures during the heating up process (Figures S29S33). We analyzed the aliquots by ${ }^{1} \mathrm{H}$ NMR and by transmission electron microscopy (TEM) to correlate the synthesis of the identified byproducts with the formation of the $\mathrm{CeO}_{2-x} \mathrm{NCs}$. We observed that at $100{ }^{\circ} \mathrm{C}$ the secondary aldimine $\mathbf{6}$ is already present, before any NCs are formed (Figure S33). The amide 9, the alkene 2, and the alcohol $\mathbf{3}$ appear only in measurable concentrations above $200{ }^{\circ} \mathrm{C}$ when the complex starts decomposing, indicated by strong gas evolution and a darkening of the solution (Figure S29). These changes happen simultaneously with the formation of the NCs, as revealed by TEM (Figure S31). As the temperature increases to $300{ }^{\circ} \mathrm{C}$, the NCs grow, but no new resonances appear in the ${ }^{1} \mathrm{H}$ NMR spectrum of the reaction mixture (Figure S33).

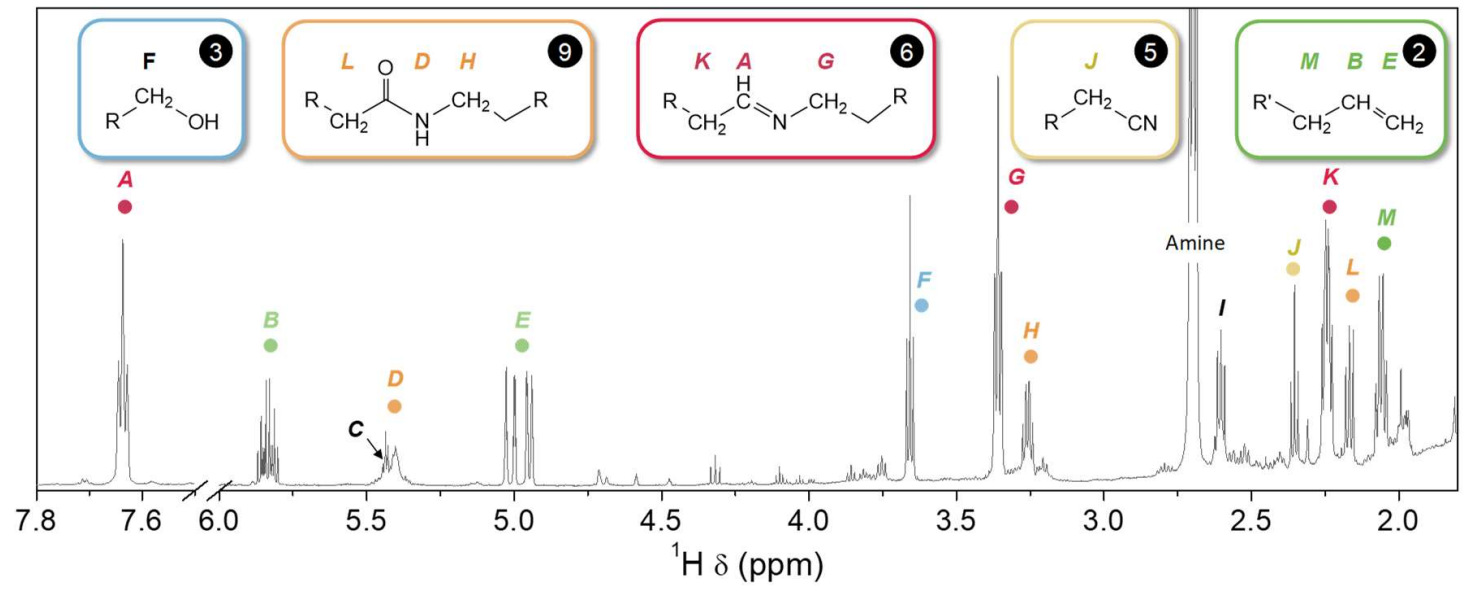

Figure 2. ${ }^{1} \mathrm{H}$ NMR spectrum of the reaction mixture of NCs prepared with hexadecylamine in octadecane after the completion of the synthesis. The most intense resonance, labeled "amine", corresponds to hexadecylamine. Resonances $C$ and $I$ could not be assigned. 
Scheme 1. Proposed reaction path for the formation of carboxylic acid through the oxidation of alkylamines by nitrate

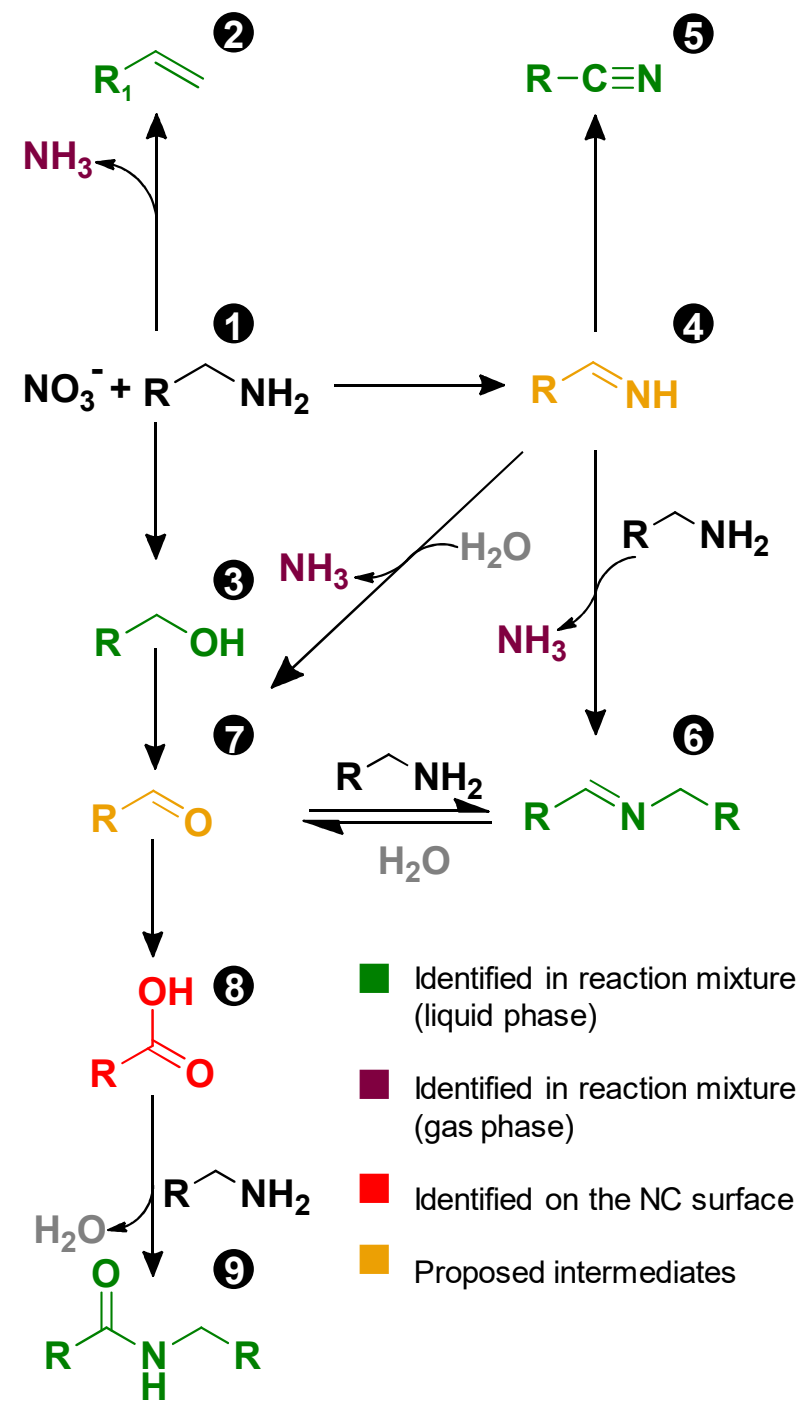

To validate the proposed reaction path, we studied the synthesis of $\mathrm{CeO}_{2-\mathrm{x}} \mathrm{NCs}$ using a secondary amine. NCs are still formed with $\mathrm{N}, \mathrm{N}$-dioctadecylamine and they have a quasispherical shape and similar size to those prepared with oleylamine (Figure S22). However, the second $\mathrm{N}$-substituent on the ligand has two effects on the reaction path. Firstly, the oxidation of the secondary amine immediately results in the stable, secondary aldimine $\mathbf{6}$. Since the primary aldimine $\mathbf{4}$ is not formed, oxidation to nitrile is prevented. Secondly, N,N-dioctadecylamine has a more basic leaving group $\left(\mathrm{pK}_{b} \mathrm{NH}_{3}>\mathrm{pK}_{\mathrm{b}} \mathrm{NH}_{2} \mathrm{R}\right)$ and more steric hindrance than hexadecylamine. This severely hinders the formation of elimination and substitution products such as alkenes and alcohols. Indeed, we observe only a significant amount of secondary aldimine $\mathbf{6}$ in the reaction mixture with N,N-dioctadecylamine (Figure S20, Table S2). Futhermore, the resulting NCs are capped by amine (Figure S21-S23, Table S3). The fact that we do not observe the fatty acid or the amide, suggests that hydrolysis of the secondary aldimine $\mathbf{6}$, is negligible under these conditions and thus that the aldehyde 7 is most likely the direct oxidation product of the alcohol $\mathbf{3}$.
Finally, to prove the generality of these results, we verified that the oxidation of amines by nitrate is not exclusive to the synthesis of $\mathrm{CeO}_{2-\mathrm{x}} \mathrm{NCs}$. We decomposed $\mathrm{Ni}, \mathrm{Zn}$ and $\mathrm{Zr}$ nitrates in the presence of alkylamines and to form $\mathrm{NiO}$ and $\mathrm{ZnO} \mathrm{NCs}$, and colloidally unstable $\mathrm{ZrO}_{2}$ particles and found that in all cases, the composition of the reaction mixture is the same as for $\mathrm{CeO}_{2-\mathrm{x}}$ and that, at least for $\mathrm{ZnO} \mathrm{NCs,} \mathrm{the} \mathrm{ligand} \mathrm{shell} \mathrm{is} \mathrm{also} \mathrm{composed} \mathrm{only} \mathrm{of} \mathrm{carboxylic}$ acid (Figures S26-S28).

While there is precedent for alkylamines showing reactivity in $\mathrm{NC}$ synthesis (besides their function as ligand), the chemistry shown here is unique. Alkylamines have been used as reducing agents, ${ }^{54,55}$ or as a source of ammonia. ${ }^{50}$ Certain side reactions were reported, such as the oxidation to nitriles, ${ }^{47,56,57}$ or the condensation with carboxylic acids. ${ }^{53}$ However, none of these previously reported transformations yielded byproducts with high binding affinity for the NC surface. In this work, we show that alkylamines are oxidized by nitrate to carboxylic acids, thus producing in situ another potential ligand. This transformation completely alters the final NC surface chemistry since the ligand shell is found only composed of carboxylate due to the higher binding affinity of carboxylates to metal oxide NCs, compared to amines. ${ }^{27}$

In summary, we scrutinized the synthesis of metal oxide NCs from metal nitrates in the presence of alkylamine ligands and revealed the oxidation of these amines with a comprehensive reaction scheme. We further proved that these reactions lead to the formation of carboxylic acids, which bind tightly to the NC surface, acting as the only capping ligand. Other NCs like metals and metal nitrides are also often synthesized from metal nitrates. Therefore, our current results might be relevant to understand these systems too.

\section{ASSOCIATED CONTENT}

\section{Supporting Information}

Chemicals used, synthesis protocols, NMR experiments supporting the identification of the compounds $\left({ }^{1} \mathrm{H}\right.$ NMR, ${ }^{13} \mathrm{C}$ NMR, COSY, HMBC, and HSQC spectra, and reference-spiking experiments) XRD, TEM, XPS measurements, and PDF analysis.

\section{AUTHOR INFORMATION}

\section{Corresponding Author}

* Maria Ibáñez: mibanez@ist.ac.at https://ibanezgroup.pages.ist.ac.at

* Jonathan De Roo: Jonathan.deroo@unibas.ch https://deroo.chemie.unibas.ch/en/

\section{Present Addresses}

\$ Institute of Energy Technologies, Department of Chemical Engineering and Barcelona Research Center in Multiscale Science and Engineering, Universitat Politecnica de Catalunya, 08019 Barcelona, Spain

$\dagger$ Department of Chemistry, University of Basel, 4058 Basel, Switzerland.

\section{ACKNOWLEDGMENT}

This work was financially supported by IST Austria and the Werner Siemens Foundation. M.C. has received funding from the European Union's Horizon 2020 research and innovation programme under the Marie Skłodowska-Curie Grant Agreement No. 665385. The work was also financially supported by University of Basel, SNSF NCCR Molecular Systems Engineering (project number: 182895) and SNSF R'equip (project number: 189622). J.L. acknowledges the MICINN/FEDER RTI2018-093996-B-C3 project. 


\section{REFERENCES}

(1) McMurtry, B. M.; Qian, K.; Teglasi, J. K.; Swarnakar, A. K.; De Roo, J.; Owen, J. S. Continuous Nucleation and Size Dependent Growth Kinetics of Indium Phosphide Nanocrystals. Chem. Mater. 2020, 32 (10), 4358-4368.

(2) Zhao, L. D.; Lo, S. H.; He, J.; Li, H.; Biswas, K.; Androulakis, J.; Wu, C. I.; Hogan, T. P.; Chung, D. Y.; Dravid, V. P.; Kanatzidis, M. G. High Performance Thermoelectrics from Earth-Abundant Materials: Enhanced Figure of Merit in PbS by Second Phase Nanostructures. J. Am. Chem. Soc. 2011, 133 (50), 20476-20487. Jansons, A. W.; Hutchison, J. E. Continuous Growth of Metal Oxide Nanocrystals: Enhanced Control of Nanocrystal Size and Radial Dopant Distribution. ACS Nano 2016, 10 (7), 6942-6951. Billet, J.; Dujardin, W.; De Keukeleere, K.; De Buysser, K.; De Roo, J.; Van Driessche, I. Size Tunable Synthesis and Surface Chemistry of Metastable $\mathrm{TiO}_{2}-$ Bronze Nanocrystals. Chem. Mater. 2018, 30 (13), 4298-4306.

(5) Berestok, T.; Guardia, P.; Blanco, J.; Nafria, R.; Torruella, P.; López-Conesa, L.; Estradé, S.; Ibáñez, M.; De Roo, J.; Luo, Z.; Cadavid, D.; Martins, J. C.; Kovalenko, M. V.; Peiró, F.; Cabot, A. Tuning Branching in Ceria Nanocrystals. Chem. Mater. 2017, 29 (10), 4418-4424.

An, K.; Kwon, S. G.; Park, M.; Na, H. Bin; Baik, S. I.; Yu, J. H.; Kim, D.; Son, J. S.; Kim, Y. W.; Song, I. C.; Moon, W. K.; Park, H. M.; Hyeon, T. Synthesis of Uniform Hollow Oxide Nanoparticles through Nanoscale Acid Etching. Nano Lett. 2008, $8(12), 4252-4258$

(7) Li, W.; Zamani, R.; Rivera Gil, P.; Pelaz, B.; Ibáñez, M.; Cadavid, D.; Shavel, A.; Alvarez-Puebla, R. A.; Parak, W. J.; Arbiol, J.; Cabot, A. CuTe Nanocrystals: Shape and Size Control, Plasmonic Properties, and Use as SERS Probes and Photothermal Agents. J. Am. Chem. Soc. 2013, 135 (19), 7098-7101.

(8) Soon, G. K.; Hyeon, T. Colloidal Chemical Synthesis and Formation Kinetics of Uniformly Sized Nanocrystals of Metals, Oxides, and Chalcogenides. Acc. Chem. Res. 2008, 41 (12), 1696-1709.

(9) Sytnyk, M.; Kirchschlager, R.; Bodnarchuk, M. I.; Primetzhofer, D.; Kriegner, D.; Enser, H.; Stangl, J.; Bauer, P.; Voith, M.; Hassel, A. W.; Krumeich, F.; Ludwig, F.; Meingast, A.; Kothleitner, G.; Kovalenko, M. V.; Heiss, W. Tuning the Magnetic Properties of Metal Oxide Nanocrystal Heterostructures by Cation Exchange. Nano Lett. 2013, 13 (2), 586-593.

(10) Liu, Y.; Cadavid, D.; Ibáñez, M.; De Roo, J.; Ortega, S.; Dobrozhan, O.; Kovalenko, M.; Cabot, A. Colloidal AgSbSe 2 Nanocrystals: Surface Analysis, Electronic Doping and Processing into Thermoelectric Nanomaterials. J. Mater. Chem. C 2016, 4 (21), 4756-4762.

(11) Yarema, O.; Yarema, M.; Wood, V. Tuning the Composition of Multicomponent Semiconductor Nanocrystals: The Case of I-IIIVI Materials. Chem. Mater. 2018, 30 (5), 1446-1461.

(12) Yin, Y.; Alivisatos, A. P. Colloidal Nanocrystal Synthesis and the Organic-Inorganic Interface. Nature 2005, 437 (7059), 664-670.

(13) Heuer-Jungemann, A.; Feliu, N.; Bakaimi, I.; Hamaly, M.; Alkilany, A.; Chakraborty, I.; Masood, A.; Casula, M. F.; Kostopoulou, A.; Oh, E.; Susumu, K.; Stewart, M. H.; Medintz, I. L.; Stratakis, E.; Parak, W. J.; Kanaras, A. G. The Role of Ligands in the Chemical Synthesis and Applications of Inorganic Nanoparticles. Chem. Rev. 2019, 119 (8), 4819-4880.

(14) De Roo, J.; Van Den Broeck, F.; De Keukeleere, K.; Martins, J. C.; Van Driessche, I.; Hens, Z. Unravelling the Surface Chemistry of Metal Oxide Nanocrystals, the Role of Acids and Bases. J. Am. Chem. Soc. 2014, 136 (27), 9650-9657.

(15) Yang, Y.; Qin, H.; Jiang, M.; Lin, L.; Fu, T.; Dai, X.; Zhang, Z.; Niu, Y.; Cao, H.; Jin, Y.; Zhao, F.; Peng, X. Entropic Ligands for Nanocrystals: From Unexpected Solution Properties to Outstanding Processability. Nano Lett. 2016, 16 (4), 2133-2138.

(16) Knowles, K. E.; Tagliazucchi, M.; Malicki, M.; Swenson, N. K.; Weiss, E. A. Electron Transfer as a Probe of the Permeability of Organic Monolayers on the Surfaces of Colloidal PbS Quantum Dots. J. Phys. Chem. C 2013, 117 (30), 15849-15857.

(17) Shi, M.; Kwon, H. S.; Peng, Z.; Elder, A.; Yang, H. Effects of Surface Chemistry on the Generation of Reactive Oxygen Species by Copper Nanoparticles. ACS Nano 2012, 6 (3), 2157-2164.
(18) Gabka, G.; Bujak, P.; Gryszel, M.; Kotwica, K.; Pron, A. Anchor Groups Effect on Spectroscopic and Electrochemical Properties of Quaternary Nanocrystals Cu-In-Zn-S Capped with Arylamine Derivatives. J. Phys. Chem. C 2015, 119 (17), 9656-9664.

(19) Irtem, E.; Arenas Esteban, D.; Duarte, M.; Choukroun, D.; Lee, S.; Ibáñez, M.; Bals, S.; Breugelmans, T. Ligand-Mode Directed Selectivity in $\mathrm{Cu}-\mathrm{Ag}$ Core-Shell Based Gas Diffusion Electrodes for CO2Electroreduction. ACS Catal. 2020, 10 (22), 1346813478 .

(20) Elimelech, O.; Aviv, O.; Oded, M.; Banin, U. A Tale of Tails: Thermodynamics of CdSe Nanocrystal Surface Ligand Exchange. Nano Lett. 2020, 20 (9), 6396-6403.

(21) Kroupa, D. M.; Vörös, M.; Brawand, N. P.; McNichols, B. W.; Miller, E. M.; Gu, J.; Nozik, A. J.; Sellinger, A.; Galli, G.; Beard, M. C. Tuning Colloidal Quantum Dot Band Edge Positions through Solution-Phase Surface Chemistry Modification. Nat. Commun. 2017, 8 (1), 1-8.

(22) Anderson, N. C.; Owen, J. S. Soluble, Chloride-Terminated CdSe Nanocrystals: Ligand Exchange Monitored by ${ }^{1} \mathrm{H}$ and ${ }^{31} \mathrm{P}$ NMR Spectroscopy. Chem. Mater. 2013, 25 (1), 69-76.

(23) Wei, J.; Schaeffer, N.; Pileni, M. P. Ag Nanocrystals: 1. Effect of Ligands on Plasmonic Properties. J. Phys. Chem. B 2014, 118 (49), 14070-14075.

(24) Ibáñez, M.; Hasler, R.; Genç, A.; Liu, Y.; Kuster, B.; Schuster, M.; Dobrozhan, O.; Cadavid, D.; Arbiol, J.; Cabot, A.; Kovalenko, M. V. Ligand-Mediated Band Engineering in Bottom-up Assembled SnTe Nanocomposites for Thermoelectric Energy Conversion. J. Am. Chem. Soc. 2019, 141 (20), 80258029.

(25) Vale, B. R. C.; Mourão, R. S.; Bettini, J.; Sousa, J. C. L.; Ferrari, J. L.; Reiss, P.; Aldakov, D.; Schiavon, M. A. Ligand Induced Switching of the Band Alignment in Aqueous Synthesized CdTe/CdS Core/Shell Nanocrystals. Sci. Reports $201991 \mathbf{2 0 1 9}$, $9(1), 1-12$.

(26) Boles, M. A.; Ling, D.; Hyeon, T.; Talapin, D. V. The Surface Science of Nanocrystals. Nat. Mater. 2016, 15 (2), 141-153.

(27) De Roo, J.; Justo, Y.; De Keukeleere, K.; Van Den Broeck, F Martins, J. C.; Van Driessche, I.; Hens, Z. Carboxylic-AcidPassivated Metal Oxide Nanocrystals: Ligand Exchange Characteristics of a New Binding Motif. Angew. Chemie - Int. Ed. 2015, 54 (22), 6488-6491.

(28) Drijvers, E.; De Roo, J.; Martins, J. C.; Infante, I.; Hens, Z. Ligand Displacement Exposes Binding Site Heterogeneity on CdSe Nanocrystal Surfaces. Chem. Mater. 2018, 30 (3), 1178 1186.

(29) Calvin, J. J.; O’Brien, E. A.; Sedlak, A. B.; Balan, A. D Alivisatos, A. P. Thermodynamics of Composition Dependent Ligand Exchange on the Surfaces of Colloidal Indium Phosphide Quantum Dots. ACS Nano 2021, 15 (1), 1407-1420.

(30) Ibáñez, M.; Korkosz, R. J.; Luo, Z.; Riba, P.; Cadavid, D.; Ortega, S.; Cabot, A.; Kanatzidis, M. G. Electron Doping in Bottom-up Engineered Thermoelectric Nanomaterials through $\mathrm{HCl}-$ Mediated Ligand Displacement. J. Am. Chem. Soc. 2015, 137 (12), 4046-4049.

(31) Liu, Y.; Gibbs, M.; Puthussery, J.; Gaik, S.; Ihly, R.; Hillhouse, H. W.; Law, M. Dependence of Carrier Mobility on Nanocrystal Size and Ligand Length in PbSe Nanocrystal Solids. Nano Lett. 2010, 10 (5), 1960-1969.

(32) Ibáñez, M.; Genç, A.; Hasler, R.; Liu, Y.; Dobrozhan, O. Nazarenko, O.; De La Mata, M.; Arbiol, J.; Cabot, A.; Kovalenko, M. V. Tuning Transport Properties in Thermoelectric Nanocomposites through Inorganic Ligands and Heterostructured Building Blocks. ACS Nano 2019, 13 (6), 6572-6580.

(33) Zeng, B.; Palui, G.; Zhang, C.; Zhan, N.; Wang, W.; Ji, X.; Chen, B.; Mattoussi, H. Characterization of the Ligand Capping of Hydrophobic CdSe-ZnS Quantum Dots Using NMR Spectroscopy. Chem. Mater. 2018, 30 (1), 225-238.

(34) Boles, M. A.; Talapin, D. V. Binary Assembly of PbS and Au Nanocrystals: Patchy PbS Surface Ligand Coverage Stabilizes the $\mathrm{CuAu}$ Superlattice. ACS Nano 2019, 13 (5), 5375-5384.

(35) Moreels, I.; Justo, Y.; De Geyter, B.; Haustraete, K.; Martins, J. C.; Hens, Z. Size-Tunable, Bright, and Stable PbS Quantum Dots: A Surface Chemistry Study. ACS Nano 2011, 5 (3), 2004-2012.

(36) Kessler, M. L.; Dempsey, J. L. Mapping the Topology of PbS Nanocrystals through Displacement Isotherms of Surface-Bound 
Metal Oleate Complexes. Chem. Mater 2020, 32, 44 Leemans, J.; Dümbgen, K. C.; Minjauw, M. M.; Zhao, Q.; Vantomme, A.; Infante, I.; Detavernier, C.; Hens, Z. Acid-Base Mediated Ligand Exchange on Near-Infrared Absorbing, IndiumBased III-V Colloidal Quantum Dots. J. Am. Chem. Soc. 2021, 143 (11), 4290-4301.

(38) De Keukeleere, K.; Coucke, S.; De Canck, E.; Van Der Voort, P.; Delpech, F.; Coppel, Y.; Hens, Z.; Van Driessche, I.; Owen, J. S.; De Roo, J. Stabilization of Colloidal $\mathrm{Ti}, \mathrm{Zr}$, and $\mathrm{Hf}$ Oxide Nanocrystals by Protonated Tri-n-Octylphosphine Oxide (TOPO) and Its Decomposition Products. Chem. Mater. 2017, 29 (23), 10233-10242.

(39) Sridaeng, D.; Limsirinawa, A.; Sirojpornphasut, P.; Chawiwannakorn, S.; Chantarasiri, N. Metal AcetylacetonateAmine and Metal Nitrate-Amine Complexes as Low-Emission Catalysts for Rigid Polyurethane Foam Preparation. J. Appl. Polym. Sci. 2015, 132 (31), 42332.

(40) Dhaene, E.; Billet, J.; Bennett, E.; Van Driessche, I.; De Roo, J. The Trouble with ODE: Polymerization during Nanocrystal Synthesis. Nano Lett. 2019, 19 (10), 7411-7417.

(41) Yu, T.; Joo, J.; Park, Y. Il; Hyeon, T. Large-Scale Nonhydrolytic Sol-Gel Synthesis of Uniform-Sized Ceria Nanocrystals with Spherical, Wire, and Tadpole Shapes. Angew. Chemie - Int. Ed. 2005, 44 (45), 7411-7414.

(42) Valdez, C. N.; Schimpf, A. M.; Gamelin, D. R.; Mayer, J. M. Low Capping Group Surface Density on Zinc Oxide Nanocrystals. ACS Nano 2014, 8 (9), 9463-9470.

(43) De Roo, J.; Yazdani, N.; Drijvers, E.; Lauria, A.; Maes, J.; Owen, J. S.; Van Driessche, I.; Niederberger, M.; Wood, V.; Martins, J. C.; Infante, I.; Hens, Z. Probing Solvent-Ligand Interactions in Colloidal Nanocrystals by the NMR Line Broadening. Chem. Mater. 2018, 30 (15), 5485-5492.

(44) Baranov, D.; Lynch, M. J.; Curtis, A. C.; Carollo, A. R.; Douglass, C. R.; Mateo-Tejada, A. M.; Jonas, D. M. Purification of Oleylamine for Materials Synthesis and Spectroscopic Diagnostics for Trans Isomers. Chem. Mater. 2019, 31 (4), 12231230.

(45) Southern, T. M.; Wendlandt, W. W. The Thermal Decomposition of Metal Complexes-XX. Some Amine Copper(II) Nitrate Complexes. J. Inorg. Nucl. Chem. 1970, 32 (12), 3783-3792.

(46) Moldoveanu, S. C. Pyrolysis of Amines and Imines. In Techniques and Instrumentation in Analytical Chemistry;
Elsevier, 2010; Vol. 28, pp 349-364.

Hiramatsu, H.; Osterloh, F. E. A Simple Large-Scale Synthesis of Nearly Monodisperse Gold and Silver Nanoparticles with Adjustable Sizes and with Exchangeable Surfactants. Chem. Mater. 2004, 16 (13), 2509-2511.

(48) Kobayashi, S.; Nagayama, S. Aldehydes vs Aldimines. Unprecedented Reactivity in Their Enolate Addition Reactions. J. Org. Chem. 1997, 62 (2), 232-233.

(49) Clayden, J.; Greeves, N.; Warren, S. Organic Chemistry, 2nd ed.; OUP Oxford, 2012

(50) Chen, Y.; Landes, N. T.; Little, D. J.; Beaulac, R. Conversion Mechanism of Soluble Alkylamide Precursors for the Synthesis of Colloidal Nitride Nanomaterials. J. Am. Chem. Soc. 2018, 140 (33), 10421-10424.

(51) Layer, R. W. The Chemistry of Imines. Chem. Rev. 1963, 63 (5), 489-510.

(52) Rawalay, S. S.; Shechter, H. Oxidation of Primary, Secondary, and Tertiary Amines with Neutral Permanganate. A Simple Method for Degrading Amines to Aldehydes and Ketones. J. Org. Chem. 1967, 32 (10), 3129-3131.

(53) De Roo, J.; Van Driessche, I.; Martins, J. C.; Hens, Z. Colloidal Metal Oxide Nanocrystal Catalysis by Sustained Chemically Driven Ligand Displacement. Nat. Mater. 2016, 15 (5), 517-521.

(54) Mourdikoudis, S.; Liz-Marzán, L. M. Oleylamine in Nanoparticle Synthesis. Chem. Mater. 2013, 25 (9), 1465-1476.

(55) Li, Z; Ji, Y; Xie, R.; Grisham, S. Y; Peng, X. Correlation of CdS Nanocrystal Formation with Elemental Sulfur Activation and Its Implication in Synthetic Development. J. Am. Chem. Soc. 2011, 133 (43), 17248-17256.

(56) Kadzutu-Sithole, R.; Machogo-Phao, L. F. E.; Kolokoto, T.; Zimuwandeyi, M.; Gqoba, S. S.; Mubiayi, K. P.; Moloto, M. J.; Van Wyk, J.; Moloto, N. Elucidating the Effect of Precursor Decomposition Time on the Structural and Optical Properties of Copper (I) Nitride Nanocubes. RSC Adv. 2020, 10 (56), 3423134246.

(57) Chen, M.; Feng, Y. G.; Wang, X.; Li, T. C.; Zhang, J. Y.; Qian, D. J. Silver Nanoparticles Capped by Oleylamine: Formation, Growth, and Self-Organization. Langmuir 2007, 23 (10), 52965304. 